\title{
ASYMPTOTIC BEHAVIOUR IN LINEAR VISCOELASTICITY
}

By

JAIME E. MUÑOZ RIVERA

National Laboratory for Scientific Computation (LNCC-CNPQ), and IMUFRJ, Rio de Janeiro, Brasil

\begin{abstract}
We study the asymptotic behaviour of the solution of the viscoelastic equation, and we prove for a bounded domain that the energy associated to this system approaches zero exponentially as time goes to infinity. Moreover, for the whole space $\mathbb{R}^{n}$ we will prove that the displacement vector field can be decomposed into two parts, solenoidal and irrotational, whose corresponding energies decay to zero uniformly as time goes to infinity with rates that depend on the regularity of the initial data.
\end{abstract}

1. Introduction. In this paper we will discuss the asymptotic behaviour of the total energy associated to the model whose solution describes the evolution of elastic waves in a homogeneous, isotropic, and viscoelastic medium. The corresponding mathematical scheme involves a system of integro-differential equations, with the integrals reflecting the memory effect. Through this work we will suppose that the specific relaxation function $\mathbf{E}$ is of the form $\mathbf{E}(t, \tau)=e(t-\tau)$; therefore, the constitutive relation for viscoelasticity assumes the expression

$$
\tilde{\sigma}=e(0) \tilde{\varepsilon}+\int_{0}^{t} g(t-\tau) \tilde{\varepsilon}(\tau) d \tau
$$

where $\tilde{\sigma}$ and $\tilde{\varepsilon}$ stand for the stress and strain tensors, respectively, and $g$ is the derivative of the specific relaxation function $e$ (see [1]). Denoting the mass density by $\rho$, the dynamic equation is written as $\rho \mathbf{u}_{t t}=\nabla \cdot \tilde{\sigma}$ where by

$$
\mathbf{u}(x, t)=\left(u_{1}(x, t), \ldots, u_{n}(x, t)\right)=y(\mathbf{x}, t)-\mathbf{x}
$$

we denote the displacement vector field associated with the motion and by $y$ the positive vector at time $t$ of the particle located at the point $\mathbf{x}$. Expressing $\tilde{\sigma}$ in terms of the displacement vector field we finally obtain

$$
\rho \mathbf{u}_{t t}-\mu \Delta \mathbf{u}-(\lambda+\mu) \nabla\{\operatorname{div} \mathbf{u}\}+\mu g * \Delta \mathbf{u}+(\lambda+\mu) h * \nabla\{\operatorname{div} \mathbf{u}\}=0 \text { in } Q
$$

subject to the initial conditions

$$
\mathbf{u}(x, 0)=\mathbf{u}_{0}(x), \quad \mathbf{u}_{t}(x, 0)=\mathbf{u}_{1}(x)
$$

Received January 16, 1992.

1991 Mathematics Subject Classification. Primary 35B40, 35L15, 73F15.

Key words and phrases. Linear viscoelasticity, energy decay rates, asymptotic behaviour.

(C) 1994 Brown University 
and boundary conditions (case of bounded medium)

$$
\mathbf{u}(x, t)=\mathbf{0} \text { on } \Sigma=\Gamma \times] 0, T[.
$$

The sign "*" denotes the convolution product in time, which is defined here as

$$
\eta * \mathbf{v}=\int_{0}^{t} \eta(t-\tau) \mathbf{v}(x, \tau) d t
$$

and by $\Omega$ we denote a bounded open set with smooth boundary $\Gamma$ or the whole space $\mathbb{R}^{n}$.

Asymptotic stability in linear viscoelasticity was studied by C. Dafermos [2] in the framework of the abstract Volterra's equation. By assuming kernels of convolution type, Dafermos concluded that the solution of this class of integro-differentiable equations is asymptotically stable provided the derivative with respect to time of the specific relaxation function is convex. Later on, this result was improved in [3] for other types of relaxation functions without the convexity assumption. Concerning the rate of decay of the solutions, the work of G. Dassios and F. Zafiropoulos [5], for homogeneous and isotropic viscoelastic materials that occupy the whole tridimensional space, establishes that the longitudinal and transverse wave decay to zero uniformly as $t^{-m-9 / 2}$, where $m$ increases depending on the symmetry of the initial datum, provided the derivative of the density relaxation is an exponential function as $t \mapsto \mu_{0} e^{-\gamma t}$. The method the authors used is based on the study of the roots of the characteristic polynomial associated with the ordinary equation, which is obtained by taking the Fourier transform of system (1.1) and then differentiating the result with respect to time. By using the fact that the kernel $g$ is an exponential function, that is, $g^{\prime}(t)=-\gamma g(t)$, the convolution term is eliminated; so the authors work with the resulting pure ordinary differential equation. To our mind, however, this method is not very efficient because the analysis of the roots is almost impossible for more general relaxation functions, even for those whose derivatives are of the form of a linear combination of exponential terms with varying rates of decay.

The main result of this paper is that the total energy associated to the viscoelastic system (1.1) decays to zero exponentially for bounded domains. For the whole space $\mathbb{R}^{n}$ the decay also holds uniformly but with algebraic rates that depend on the regularity of the initial data. More precisely, if the initial datum is the $m$ th derivative of a function in $L^{1}\left(\mathbb{R}^{n}\right)$, then the energy decays as $t^{-m-n / 2}$ when time goes to infinity, provided the following conditions hold:

$$
\begin{gathered}
h=g, \quad h, g>0 \\
g^{\prime}(t) \leq-c g(t) \quad \text { and } \quad g^{\prime \prime}(t) \leq C g(t) \\
g^{\prime}(t) \geq-\kappa g(t)
\end{gathered}
$$

where $\kappa, c, C$ are positive constants. For the case $\Omega=\mathbb{R}^{n}$, assumption (1.3) is not necessary, which means that the functions $h$ and $g$ can be chosen as any linearly independent functions; nevertheless, we must import the additional condition:

$$
h^{\prime}(t) \leq-c_{0} h(t) \quad \text { and } \quad h^{\prime \prime}(t) \leq C_{0} h(t),
$$




$$
h^{\prime}(t) \geq-\kappa_{0} h(t)
$$

where $\kappa_{0}, c_{0}, C_{0}$ are positive constants.

Moreover, we will prove that the displacement vector field can be decomposed into two parts, solenoidal and irrotational, whose associated energy decays to zero as indicated in Theorem 4.1 of Sec. 4.

Note that $g$ and $h$ can be a linear combination of exponential terms with varying rates of decay. In general $g$ and $h$ can be functions of the form $\sum \rho_{i}(t) e^{-\lambda_{i} t}$ where $\rho_{i}$ is a nonnegative polynomial function such that $\rho_{i}^{\prime} \leq 0$.

Here we use basically the energy method in order to construct a suitable Liapunov functional whose derivative is negatively proportional to itself. This property will prove that the functional decays to zero exponentially. Regularity results and some technical ideas are also used. In Sec. 2 we will establish the existence and regularity results, while Secs. 3 and 4 are devoted to the study of the asymptotic behaviour of the energy for bounded domains and the whole space $\mathbb{R}^{n}$, respectively.

2. Existence, uniqueness, and regularity. In this section we will prove the existence, uniqueness, and regularity of solutions of the viscoelastic system given by Eq. (1.1). Through this section $\Omega$ will denote either a bounded domain or the whole space $\mathbb{R}^{n}$. So, to show the mean result of this paper we will introduce the following notation. Let us denote by $A$ the operator defined on a subset of $\left[L^{2}(\Omega)\right]^{n}$ given by

$$
\begin{aligned}
D(A) & =\left[H_{0}^{1}(\Omega) \cap H^{2}(\Omega)\right]^{n}, \\
A \mathbf{w} & =-\mu \Delta \mathbf{w}-(\lambda+\mu) \nabla \operatorname{div} \mathbf{w}
\end{aligned}
$$

where $\mathbf{w}=\left(w_{1}, \ldots, w_{n}\right) ;$ so for $\Delta \mathbf{w}$ we denote

$$
\Delta \mathbf{w}=\left(\Delta w_{1}, \ldots, \Delta w_{n}\right) .
$$

Finally, div stands for the divergence operator. It is well known that $A$ is a selfadjoint, positive-definite operator of $\left[L^{2}(\Omega)\right]^{n}$.

In order to facilitate our analysis we denote by “ $\square$ ” the binary function given by

$$
\{g \square f\}(t)=\int_{0}^{t} g(t-\tau)|f(t)-f(\tau)|^{2} d \tau
$$

where $f$ can be a real, complex, or vectorial function. When $f$ is either a vector of $\mathbb{R}^{n}$ or a complex number, by $|\cdot|$ we denote both the Euclidean norm of $\mathbb{R}^{n}$ or $\mathbb{C}$, respectively. Note that the sign of $g \square f$ depends solely on the sign of $g$.

REMARK 2.1. Let $\eta, \phi$, and $\psi$ be $C^{1}$-functions; then the following expressions hold:

$$
\begin{array}{r}
2(\eta * \phi) \phi^{\prime}=-\eta(t)|\phi|^{2}-\frac{d}{d t}\left\{\eta \square \phi-\left(\int_{0}^{t} \eta d \tau\right)|\phi|^{2}\right\}+\eta^{\prime} \square \phi, \\
\int_{0}^{t} \eta(t-\tau)\{\phi(\tau)-\phi(t)\} \psi(t) d \tau \leq \varepsilon|\psi(t)|^{2}+\frac{1}{4 \varepsilon}\left\{\int_{0}^{t}|\eta(\tau)| d \tau\right\} \eta \square \phi .
\end{array}
$$


In fact, a simple calculation yields

$$
\begin{aligned}
& \frac{d}{d t} \int_{0}^{t} \eta(t-\tau)|\phi(t)-\phi(\tau)|^{2} d \tau \\
& \quad=\int_{0}^{t} \eta^{\prime}(t-\tau)|\phi(t)-\phi(\tau)|^{2} d \tau \\
& \quad-2 \int_{0}^{t} \eta(t-\tau) \phi^{\prime}(t) \phi(\tau) d \tau+\left\{\int_{0}^{t} \eta(t-\tau) d \tau\right\} \frac{d}{d t}|\phi(t)|^{2}
\end{aligned}
$$

from which it follows that

$$
\begin{aligned}
2 \int_{0}^{t} \eta(t-\tau) \phi^{\prime}(t) \phi(\tau) d \tau \\
=-\frac{d}{d t}\left\{\int_{0}^{t} \eta(t-\tau)|\phi(t)-\phi(\tau)|^{2} d \tau-\int_{0}^{t} \eta(t-\tau) d \tau|\phi(t)|^{2}\right\} \\
\quad-\eta(t)|\phi(t)|^{2}+\int_{0}^{t} \eta^{\prime}(t-\tau)|\phi(t)-\phi(\tau)|^{2} d \tau
\end{aligned}
$$

which yields Eq. (2.1). Finally, by using the Hölder inequality we obtain

$$
\begin{aligned}
& \int_{0}^{t} \eta(t-\tau)\{\phi(\tau)-\phi(t)\} \psi(t) d \tau \\
& \leq|\psi(t)|\left\{\int_{0}^{t}|\eta(\tau)| d \tau\right\}^{1 / 2}\left\{\int_{0}^{t}|\eta(t-\tau)||\phi(\tau)-\phi(t)|^{2} d \tau\right\}^{1 / 2} \\
& \quad \leq \varepsilon|\psi(t)|^{2}+\frac{1}{4 \varepsilon}\left\{\int_{0}^{t}|\eta(\tau)| d \tau\right\}\left\{\int_{0}^{t}|\eta(t-\tau)||\phi(\tau)-\phi(t)|^{2} d \tau\right\},
\end{aligned}
$$

which proves the validity of Eq. (2.2).

In order to simplify notation we shall omit the variables $t, x$, or $\xi$ of the functions under the integral sign.

Theorem 2.1. Let us take $\left(\mathbf{u}_{0}, \mathbf{u}_{1}\right)$ in $D(A) \times\left[H_{0}^{1}(\Omega)\right]^{n}$, and let us suppose that $g$ and $h$ are positive $C^{2}$-functions satisfying

$$
\alpha \stackrel{\text { def }}{=} 1-\int_{0}^{\infty} g d \tau>0, \quad 1-\int_{0}^{\infty} h d \tau>0 .
$$

Under this condition there exists only one solution of system (1.1) satisfying

$$
\mathbf{u} \in C([0, T] ; D(A)) \cap C^{1}\left([0, T] ;\left[H_{0}^{1}(\Omega)\right]^{n}\right) \cap C^{2}\left([0, T] ;\left[L^{2}(\Omega)\right]^{n}\right) .
$$

Moreover, if we take $\mathbf{u}_{0} \in D\left(A^{2}\right)$ and $\mathbf{u}_{1} \in D(A)$, then the function of system (1.1) satisfies

$$
\mathbf{u} \in C^{1}([0, T] ; D(A)) \cap C^{2}\left([0, T] ;\left[H_{0}^{1}(\Omega)\right]^{n}\right) \cap C^{3}\left([0, T] ;\left[L^{2}(\Omega)\right]^{n}\right) .
$$


Proof. For bounded domains the result follows immediately from Theorem 2.2 of [3]. For the whole space $\mathbb{R}^{n}$, Theorem 2.3 of [2] shows that there exists only one solution $\mathbf{u}$ of system (1.1) satisfying

$$
\mathbf{u} \in C^{1}\left([0, T] ;\left[H_{0}^{1}(\Omega)\right]^{n}\right) \cap C^{2}\left([0, T] ;\left[L^{2}(\Omega)\right]^{n}\right) .
$$

By taking the Fourier transform, Eq. (1.1) becomes

$$
\begin{gathered}
\rho \hat{\mathbf{u}}_{t t}+\mu|\xi|^{2} \hat{\mathbf{u}}+(\lambda+\mu) \xi \sum_{i=1}^{n} \xi_{i} \hat{\mathbf{u}}_{i}+|\xi|^{2} g * \hat{\mathbf{u}}+\xi \sum_{i=1}^{n} \xi_{i} h * \hat{u}_{i}=0, \\
\hat{\mathbf{u}}(x, 0)=\hat{\mathbf{u}}_{0}(x), \quad \hat{\mathbf{u}}_{t}(x, 0)=\hat{\mathbf{u}}_{1}(x) .
\end{gathered}
$$

We next multiply the above equation by $\overline{\hat{\mathbf{u}}}_{t}$ to obtain

$$
\begin{aligned}
\frac{1}{2} \frac{d}{d t}\left\{\rho\left|\hat{\mathbf{u}}_{t}\right|^{2}+\mu \sum_{i=1}^{n}|\xi|^{2}|\hat{\mathbf{u}}|^{2}+(\lambda+\mu)\left|\sum_{i=1}^{n} \xi_{i} \hat{u}_{i}\right|^{2}\right\} \\
=|\xi|^{2} g * \hat{\mathbf{u}} \cdot \hat{\mathbf{u}}_{t}+\left\{h *\left(\sum_{i=1}^{n} \xi_{i} \hat{u}_{i}\right)\right\}\left\{\sum_{i=1}^{n} \xi_{i} \hat{u}_{i, t}\right\} .
\end{aligned}
$$

By applying Remark 2.1 we get

$$
\begin{aligned}
& \frac{1}{2} \frac{d}{d t}\left\{\rho\left|\hat{\mathbf{u}}_{t}\right|^{2}+\sum_{i=1}^{n}\left(\mu-\int_{0}^{t} g d \tau\right)|\xi|^{2}|\hat{\mathbf{u}}|^{2}+\left(\lambda+\mu-\int_{0}^{t} h d \tau\right)\left|\sum_{i=1}^{n} \xi_{i} \hat{u}_{i}\right|^{2}\right\} \\
& \quad+\frac{1}{2} \frac{d}{d t}\left\{|\xi|^{2} g \square \hat{\mathbf{u}}+\frac{1}{2} h \square \sum_{i=1}^{n} \xi_{i} \hat{u}_{i}\right\}=-\frac{1}{2}|\xi|^{2} g^{\prime} \square \hat{\mathbf{u}}-\frac{1}{2} h^{\prime} \square \sum_{i=1}^{n} \xi_{i} \hat{u}_{i} .
\end{aligned}
$$

Since $g$ and $h$ are $C^{1}$-functions satisfying $g(t) \geq g_{0}>0, h(t) \geq h_{0}>0$ on $[0, T]$, we conclude that there exists $c>0$ such that

$$
\frac{d}{d t} E(\xi, t) \leq c E(\xi, t)
$$

where

$$
\begin{aligned}
E(\xi, t)= & \rho\left|\hat{\mathbf{u}}_{t}\right|^{2}+\sum_{i=1}^{n}\left(\mu-\int_{0}^{t} g d \tau\right)|\xi|^{2}|\hat{\mathbf{u}}|^{2} \\
& +\left(\lambda+\mu-\int_{0}^{t} h d \tau\right)\left|\sum_{i=1}^{n} \xi_{i} \hat{u}_{i}\right|^{2}+|\xi|^{2} g \square \hat{\mathbf{u}}+\frac{1}{2} h \square \sum_{i=1}^{n} \xi_{i} \hat{u}_{i} ;
\end{aligned}
$$

therefore, Gronwall's inequality implies

$$
E(\xi, t) \leq E(\xi, 0) e^{c t} .
$$


Multiplying the above inequality by $\left(1+|\xi|^{2}\right)^{m}$ and integrating in $\mathbb{R}^{n}$ for $m=1,3$, we conclude

$$
\mathbf{u} \in C\left(0, T ; D\left(A^{2}\right)\right) \quad \text { and } \quad \mathbf{u}_{t} \in C(0, T ; D(A))
$$

which together with Eq. (1.1) establishes Eq. (2.5). The proof is now complete.

The study of the asymptotic behaviour is a rather complicated task, which will be the object of the following section.

3. Asymptotic behaviour. Our objective in this section is to show that the total energy associated to the viscoelastic system on a bounded smooth region decays to zero exponentially provided conditions (1.3)-(1.5) and (2.3) hold. From now on and without loss of generality we will suppose that $\rho=1$.

In order to prove the asymptotic behaviour of the energy we will introduce the following functions:

$$
\begin{aligned}
E_{1}(t) \stackrel{\text { def }}{=} & \frac{\mu}{2} \sum_{i=1}^{n} \int_{\Omega} g \square \nabla u_{i}+\frac{1}{2}(\mu+\lambda) \int_{\Omega} g \square \operatorname{div} \mathbf{u} d x \\
& +\frac{1}{2} \int_{\Omega}\left|\mathbf{u}_{t}\right|^{2} d x+ \\
& \frac{1}{2}\left(1-\int_{0}^{t} g d \tau\right) \\
& \times\left\{\mu \sum_{i=1}^{n} \int_{\Omega}\left|\nabla u_{i}\right|^{2} d x+(\lambda+\mu) \int_{\Omega}|\operatorname{div} \mathbf{u}|^{2} d x\right\}, \\
E_{2}(t) \stackrel{\text { def }}{=} & \frac{1}{2} \int_{\Omega} g \square A \mathbf{u}+\frac{1}{2} \sum_{i=1}^{n} \mu \int_{\Omega}\left|\nabla u_{i, t}\right|^{2} d x \\
& +\frac{1}{2}(\lambda+\mu) \int_{\Omega}\left|\operatorname{div} \mathbf{u}_{t}\right|^{2} d x+\frac{1}{2}\left(1-\int_{0}^{t} g d \tau\right) \int_{\Omega}|A \mathbf{u}|^{2} d x, \\
E_{3}(t) \stackrel{\text { def }}{=} & \frac{1}{2} \int_{\Omega}\left|\mathbf{u}_{t t}\right|^{2} d x+\frac{1}{2} \sum_{i=1}^{n} \int_{\Omega} \mu\left|\nabla u_{i, t}\right|^{2} d x+\frac{1}{2}(\mu+\lambda) \int_{\Omega}\left|\operatorname{div} \mathbf{u}_{t}\right|^{2} d x, \\
I(t) \stackrel{\text { def }}{=} & \int_{\Omega} \mathbf{u}_{t t} \cdot \mathbf{u}_{t} d x+\frac{1}{2} g(0) \int_{\Omega}\left|\mathbf{u}_{t}\right|^{2} d x \\
& +\frac{1}{2} \sum_{i=1}^{n} \int_{\Omega} \mu\left\{g(0) g+g^{\prime}\right\} \square \nabla u_{i} d x+\frac{1}{2}(\lambda+\mu) \int_{\Omega}\left\{g(0) g+g^{\prime}\right\} \square \operatorname{div} \mathbf{u} d x \\
& -\frac{1}{2}\left(\int_{0}^{t} g(0) g+g^{\prime} d \tau\right)\left(\sum_{i=1}^{n} \int_{\Omega} \mu\left|\nabla u_{i}\right|^{2} d x+(\lambda+\mu) \int_{\Omega}|\operatorname{div} \mathbf{u}|^{2} d x\right),
\end{aligned}
$$

and

$$
J(t) \stackrel{\text { def }}{=} \int_{\Omega} \mathbf{u}_{t} \cdot A \mathbf{u} d x
$$

In the following lemma we establish an inequality which will play an important role in obtaining our decay result. To do this we will take advantage of the kernel properties in Eqs. (1.4) and (1.5) which cause the memory term to have a damping effect. 
Lemma 3.1. Let us suppose that $g \in C^{2}$ satisfies Eqs. (1.3)-(1.5); then for any initial datum $\left(\mathbf{u}_{0}, \mathbf{u}_{1}\right)$ taken in $D\left(A^{2}\right) \times D(A)$ we have

$$
\begin{aligned}
\frac{d}{d t}\left\{E_{3}(t)\right. & \left.+g(0)\left\{1-\frac{g(0)}{4 \kappa}\right\} I(t)+g(0)\left\{1-\frac{g(0)}{2 \kappa}\right\} J(t)\right\} \\
\leq & -\alpha \frac{g(0)^{2}}{4 \kappa} \int_{\Omega}\left\{\left|\mathbf{u}_{t t}\right|^{2}+\mathbf{u}_{t} \cdot A \mathbf{u}_{t}+|A \mathbf{u}|^{2}\right\} d x \\
& +\left\{\frac{4 \kappa}{g(0)(1-\alpha)}+\frac{4 \kappa}{\alpha}\right\} \int_{\Omega} g \square A \mathbf{u} d x \\
& +\kappa g(0) g(t) \int_{\Omega} \mathbf{u} \cdot A \mathbf{u} d x+\frac{\mu}{2} C g(0) \sum_{i=1}^{n} \int_{\Omega} g \square \nabla u_{i} d x \\
& +\frac{1}{2} C g(0)(\lambda+\mu) \int_{\Omega} g \square \operatorname{div} \mathbf{u} d x .
\end{aligned}
$$

Proof. Differentiating system (2.2) with respect to time and then substituting $A \mathbf{u}$ given by Eq. (1.1) yields

$$
\mathbf{u}_{t t t}+A \mathbf{u}_{t}+g(0) \mathbf{u}_{t t}-f * A \mathbf{u}=0
$$

where $f=g(0) g+g^{\prime}$. Multiplying by $\mathbf{u}_{t t}$ and integrating in $\Omega$ we have

$$
\frac{1}{2} \frac{d}{d t}\left\{\int_{\Omega}\left|\mathbf{u}_{t t}\right|^{2}+\int_{\Omega} \mathbf{u}_{t} \cdot A \mathbf{u}_{t} d x\right\}=-g(0) \int_{\Omega}\left|\mathbf{u}_{t t}\right|^{2} d x+\int_{\Omega} f * A \mathbf{u} \cdot \mathbf{u}_{t t} d x
$$

Inserting the identity

$$
f * A \mathbf{u}=\left\{\int_{0}^{t} f(\tau) d \tau\right\} A \mathbf{u}+\int_{0}^{t} f(t-\tau)\{A \mathbf{u}(x, \tau)-A \mathbf{u}(x, t)\} d \tau
$$

into Eq. (3.3) we get

$$
\begin{aligned}
\frac{d}{d t} E_{3}^{m}(t)= & -g(0) \int_{\Omega}\left|\mathbf{u}_{t t}\right|^{2} d x+\left\{\int_{0}^{t} f d \tau\right\} \int_{\Omega} \mathbf{u}_{t t} \cdot A \mathbf{u} d x \\
& +\int_{\Omega} \int_{0}^{t} f(t-\tau)\{A \mathbf{u}(x, \tau)-A \mathbf{u}(x, t)\} \cdot \mathbf{u}_{t t} d \tau d x .
\end{aligned}
$$

Substituting $\mathbf{u}_{t t}$ given by Eq. (1.1) into the expression $\int_{\Omega} \mathbf{u}_{t t} \cdot A \mathbf{u} d x$ yields

$$
\begin{aligned}
\int_{\Omega} \mathbf{u}_{t t} \cdot A \mathbf{u} d x= & -\left\{1-\int_{0}^{t} g(\tau) d \tau\right\} \int_{\Omega}|A \mathbf{u}|^{2} d x \\
& +\int_{\Omega} \int_{0}^{t} f(t-\tau)\{A \mathbf{u}(x, \tau)-A \mathbf{u}(x, t)\} \cdot A \mathbf{u}(x, t) d \tau d x .
\end{aligned}
$$

In view of the above identity we may rewrite Eq. (3.4) in the form

$$
\begin{aligned}
\frac{d}{d t} E_{3}^{m}(t)= & -g(0) \int_{\Omega}\left|\mathbf{u}_{t t}\right|^{2} d x-\left\{\int_{0}^{t} f d \tau\right\}\left\{1-\int_{0}^{t} g d \tau\right\} \int_{\Omega}|A \mathbf{u}|^{2} d x \\
& +\left\{\int_{0}^{t} f(\tau) d \tau\right\} \int_{\Omega} \int_{0}^{t} g(t-\tau)\{A \mathbf{u}(x, \tau)-A \mathbf{u}(x, t)\} \cdot A \mathbf{u}(x, t) d \tau d x \\
& +\int_{\Omega} \int_{0}^{t} f(t-\tau)\{A \mathbf{u}(x, \tau)-A \mathbf{u}(x, t)\} \cdot \mathbf{u}_{t t}(x, t) d \tau d x
\end{aligned}
$$


To evaluate the derivative of $I$, let us multiply Eq. (3.2) by $\mathbf{u}_{t}$ in Eq. (3.2). Integrating over $\Omega$ yields

$$
\begin{aligned}
& \frac{d}{d t}\left\{\int_{\Omega} \mathbf{u}_{t t} \cdot \mathbf{u}_{t} d x+\frac{1}{2} g(0) \int_{\Omega}\left|\mathbf{u}_{t}\right|^{2} d x\right\} \\
& \quad=\int_{\Omega}\left|\mathbf{u}_{t t}\right|^{2} d x-\int_{\Omega} A \mathbf{u}_{t} \cdot \mathbf{u}_{t} d x+\int_{\Omega}\{f * A \mathbf{u}\} \cdot \mathbf{u}_{t} d x .
\end{aligned}
$$

Using identity (2.1) we can establish

$$
\begin{aligned}
\int_{\Omega}\{f * A \mathbf{u}\} \cdot \mathbf{u}_{t} d x= & -\frac{1}{2} f(t) \int_{\Omega} A \mathbf{u} \cdot \mathbf{u} d x \\
& +\frac{1}{2} \sum_{i=1}^{n} \int_{\Omega} \mu f^{\prime} \square \nabla u_{i} d x+\frac{1}{2}(\lambda+\mu) \int_{\Omega} f^{\prime} \square \operatorname{div} \mathbf{u} d x \\
& -\frac{1}{2} \frac{d}{d t}\left\{\sum_{i=1}^{n} \int_{\Omega} \mu f \square \nabla u_{i} d x+(\lambda+\mu) \int_{\Omega} f \square \operatorname{div} \mathbf{u} d x d x\right\} \\
& +\frac{1}{2} \frac{d}{d t}\left\{\int_{0}^{t} f d \tau\right\}\left(\int_{\Omega} A \mathbf{u} \cdot \mathbf{u} d x\right) .
\end{aligned}
$$

Substituting this expression into Eq. (2.6) and recalling the definition of $I$, one has

$$
\begin{aligned}
\frac{d}{d t} I(t)= & \int_{\Omega}\left|\mathbf{u}_{t t}\right|^{2} d x-\frac{1}{2} f(t) \int_{\Omega} A \mathbf{u} \cdot \mathbf{u} d x d x \\
& -\int_{\Omega} A \mathbf{u}_{t} \cdot \mathbf{u}_{t} d x+\frac{1}{2} \sum_{i=1}^{n} \int_{\Omega} \mu f^{\prime} \square \nabla u_{i} d x+\frac{1}{2}(\lambda+\mu) \int_{\Omega} f^{\prime} \square \operatorname{div} \mathbf{u} d x .
\end{aligned}
$$

Differentiating $J$ and using Eq. (1.1) we get

$$
\begin{aligned}
\frac{d}{d t} J(t)= & \int_{\Omega} A \mathbf{u}_{t} \cdot \mathbf{u}_{t} d x-\left\{1-\int_{0}^{t} g(\tau) d \tau\right\} \int_{\Omega}|A \mathbf{u}|^{2} d x \\
& +\int_{\Omega} \int_{0}^{t} g(t-\tau)\{A \mathbf{u}(x, \tau)-A \mathbf{u}(x, t)\} \cdot A \mathbf{u}(x, t) d \tau d x
\end{aligned}
$$


which together with Eqs. (3.5) and (3.7) yields

$$
\begin{aligned}
\frac{d}{d t}\left\{E_{3}(t)\right. & \left.+g(0)\left\{1-\frac{g(0)}{4 \kappa}\right\} I(t)+g(0)\left\{1-\frac{g(0)}{2 \kappa}\right\} J(t)\right\} \\
= & -\frac{g(0)^{2}}{4 \kappa} \int_{\Omega}\left|\mathbf{u}_{t t}\right|^{2} d x-\left\{g(0)\left\{1-\frac{g(0)}{2 \kappa}\right\}+\int_{0}^{t} f d \tau\right\}\left\{1-\int_{0}^{t} g d \tau\right\} \int_{\Omega}|A \mathbf{u}|^{2} d x \\
& +\left\{g(0)\left\{1-\frac{g(0)}{2 \kappa}\right\}+\int_{0}^{t} f d \tau\right\} \int_{0}^{L} \int_{0}^{t} g(t-\tau)\{A \mathbf{u}(x, \tau)-A \mathbf{u}(x, t)\} \\
& +A \mathbf{u}(x, t) d \tau d x \\
& +\int_{\Omega} \int_{0}^{t} f(t-\tau)\{A \mathbf{u}(x, \tau)-A \mathbf{u}(x, t)\} \cdot \mathbf{u}_{t t}(x, t) d \tau d x \\
& -\frac{g(0)}{2}\left\{1-\frac{g(0)}{4 \kappa}\right\} f(t) \int_{\Omega} A \mathbf{u} \cdot \mathbf{u} d x \\
& +\frac{1}{2} g(0)\left\{1-\frac{g(0)}{4 \kappa}\right\} \sum_{i=1}^{n} \int_{\Omega} \mu f^{\prime} \square \nabla u_{i} d x \\
& +\frac{1}{2}(\lambda+\mu) g(0)\left\{1-\frac{g(0)}{4 \kappa}\right\} \int_{\Omega} f^{\prime} \square \operatorname{div} \mathbf{u} d x .
\end{aligned}
$$

On the other hand, hypotheses (1.5) and (2.3) imply that

$$
\frac{g(0)}{\kappa} \leq 1-\alpha<1 \text { and } 1-\int_{0}^{t} g d \tau \leq 1-\frac{g(0)}{\kappa}+\frac{g(t)}{\kappa}
$$

therefore, we get

$$
\frac{g(0)^{2}}{2 \kappa} \leq \int_{0}^{t} f d \tau+g(0)\left\{1-\frac{g(0)}{2 \kappa}\right\} \leq 2 g(0) .
$$

Using Eqs. (1.4) and (1.5) and the inequalities

$$
\begin{aligned}
& \left\{g(0)\left\{1-\frac{g(0)}{2 \kappa}\right\}+\int_{0}^{t} f(\tau) d \tau\right\} \\
& \quad \times \int_{0}^{L} \int_{0}^{t} g(t-\tau)\{A \mathbf{u}(x, \tau)-A \mathbf{u}(x, t)\} \cdot A \mathbf{u}(x, t) d \tau d x \\
& \quad \leq \alpha \frac{g(0)^{2}}{4 \kappa} \int_{0}^{L}|A \mathbf{u}|^{2} d x+4 \frac{\kappa}{\alpha} \int_{0}^{L} g \square A \mathbf{u} d x, \\
& \int_{\Omega} \int_{0}^{t} f(t-\tau)\{A \mathbf{u}(x, \tau)-A \mathbf{u}(x, t)\} \cdot \mathbf{u}_{t t}(x, t) d \tau d x \\
& \leq(1-\alpha) \frac{g(0)^{2}}{4 \kappa} \int_{0}^{L}\left|\mathbf{u}_{t t}\right|^{2} d x+\frac{4 \kappa}{g(0)(1-\alpha)} \int_{0}^{L} g \square A \mathbf{u} d x,
\end{aligned}
$$

together with Eq. (3.9) our result follows.

THEOREM 3.1. Under the same hypotheses of Lemma 3.1, for any initial datum $\left(\mathbf{u}_{0}, \mathbf{u}_{1}\right)$ taken in $D(A) \times\left[H_{0}^{1}(\Omega)\right]^{n}$ the corresponding solution of Eq. (1.1) satisfies

$$
E_{1}(t)+E_{2}(t)+E_{3}(t) \leq C e^{-\gamma t} \text {. }
$$


Proof. We will suppose that $\left(\mathbf{u}_{0}, \mathbf{u}_{1}\right) \in D\left(A^{2}\right) \times D(A)$; our conclusion will follow using density arguments. Using Eq. (1.1) we can show

$$
\begin{aligned}
\frac{1}{2} \frac{d}{d t} \int_{\Omega} & \left\{\left|\mathbf{u}_{t}\right|^{2}+\sum_{i=1}^{n} \mu\left|\nabla u_{i}\right|^{2}+(\lambda+\mu)|\operatorname{div} \mathbf{u}|^{2}\right\} d x \\
= & \mu \sum_{i=1}^{n} \int_{0}^{t} \int_{\Omega} g(t-\tau) \nabla u_{i}(x, \tau) \cdot \nabla u_{i, t}(x, t) d x d \tau \\
& +(\mu+\lambda) \int_{0}^{t} \int_{\Omega} g(t-\tau) \operatorname{div} u(x, \tau) \cdot \operatorname{div} u_{t}(x, t) d x d \tau
\end{aligned}
$$

Applying identity (2.1) with hypotheses (1.4), (1.5) and recalling the definition of $E_{1}$ we obtain

$$
\frac{d}{d t} E_{1}(t) \leq-\frac{1}{2} g(t) \int_{\Omega} A \mathbf{u} \cdot \mathbf{u} d x-\frac{c}{2} \mu \sum_{i=1}^{n} \int_{\Omega} g \square \nabla u_{i} d x-\frac{c}{2}(\lambda+\mu) \int_{\Omega} g \square \operatorname{div} u d x .
$$

Similarly, using Eq. (1.1) and applying Green's formulas the following identity is established:

$$
\frac{1}{2} \frac{d}{d t}\left\{\int_{\Omega} A \mathbf{u}_{t} \cdot \mathbf{u}_{t}+|A \mathbf{u}|^{2}\right\} d x=\sum_{i=1}^{n} \int_{0}^{t} \int_{\Omega} g(t-\tau) A \mathbf{u}(x, t) \cdot A \mathbf{u}_{t}(x, \tau) d x d \tau .
$$

Applying inequality (2.2) for $\eta=g, \phi=\psi=A \mathbf{u}$, and hypothesis (1.4) turns the above identity into

$$
\frac{d}{d t} E_{2}(t) \leq-\frac{c}{2} \int_{\Omega} g \square A \mathbf{u} d x .
$$

Let us introduce the following functions:

$$
\begin{aligned}
K(t)= & E_{3}(t)+g(0)\left\{1-\frac{g(0)}{4 \kappa}\right\} I(t)+g(0)\left\{1-\frac{g(0)}{2 \kappa}\right\} J(t)+N_{1} E_{1}(t)+N_{2} E_{2}(t), \\
\mathscr{N}(t)= & \int_{\Omega}\left\{\left|\mathbf{u}_{t t}\right|^{2}+\mathbf{u}_{t} \cdot A \mathbf{u}_{t}+|A \mathbf{u}|^{2}+g \square A \mathbf{u}\right\} d x \\
& +\sum_{i=1}^{n} \int_{\Omega} \mu g \square \nabla u_{i} d x+(\lambda+\mu) \int_{\Omega} g \square \operatorname{div} \mathbf{u} d x .
\end{aligned}
$$

Lemma 3.1 and relations (3.10), (3.11) imply that the inequalities

$$
\begin{aligned}
& \frac{d}{d t} K(t) \leq-\alpha \frac{g(0)^{2}}{4 \kappa} \mathscr{N}(t), \\
& c_{0} \mathscr{N}(t) \leq K(t) \leq c_{1} \mathscr{N}(t)
\end{aligned}
$$

hold for

$$
\begin{aligned}
& N_{1}=\frac{2}{c}\left\{g(0)[c \kappa+c+2 C(\mu+\lambda)]+c \frac{\kappa}{\alpha}+\frac{\alpha g(0)^{2}}{4 \kappa}\right\}, \\
& N_{2}=\frac{2}{c}\left\{\frac{4 \kappa}{g(0)(1-\alpha)}+4 \frac{\kappa}{\alpha}+c g(0)+\frac{\alpha g(0)^{2}}{4 \kappa}\right\},
\end{aligned}
$$




$$
c_{0}=\min \left\{\frac{1}{4}, g(0)\right\}, \quad c_{1}>0 .
$$

Using inequalities (3.12)-(3.17) we get

$$
\frac{d}{d t} K_{i}(t)+\gamma K_{i}(t) \leq 0
$$

for $\gamma=\alpha \frac{g(0)^{2}}{4 \kappa c_{1}}$, which implies that

$$
K(t) \leq K(0) e^{-\gamma t}
$$

Finally from Eq. (3.13) our result follows.

4. Decomposition of the displacement vector field. Here we will study the asymptotic behaviour of the energy in the whole space $\mathbb{R}^{n}$. We will prove that the displacement vector field can be decomposed into two parts: solenoidal and irrotational, whose corresponding energies decay uniformly to zero at a rate that depends on the regularity of the initial datum.

In order to assist the reader we will show the conditions for which such a decomposition holds for any vector field of $\mathbb{R}^{n}$. Let us denote $\alpha=\left(\alpha_{1}, \ldots, \alpha_{n}\right) \in \mathbb{N}^{n}$, $\mathbf{x}=\left(x_{1}, \ldots, x_{n}\right) \in \mathbb{R}^{n}$ and

$$
|\alpha|=\sum_{i=1}^{n} \alpha_{i}, \quad \mathbf{x}^{\alpha}=x_{1}^{\alpha_{1}} x_{2}^{\alpha_{2}} \cdots x_{n}^{\alpha_{n}}, \quad \partial^{\alpha}=\left(\frac{\partial}{\partial x_{1}}\right)^{\alpha_{1}} \cdots\left(\frac{\partial}{\partial x_{n}}\right)^{\alpha_{n}} .
$$

Let us define by $U(x)$ the function

$$
U(x)= \begin{cases}\frac{1}{2 \pi} \ln |x| & \text { if } n=2 \\ \frac{1}{(n-2) \sigma_{n}|x|^{n-2}} & \text { if } n>2\end{cases}
$$

where $\sigma_{n}$ is the area of the unitary ball of $\mathbb{R}^{n}$. It is well known that the solution of the equation

$$
\Delta u=f \quad \text { in } \mathbb{R}^{n}
$$

is given by

$$
u(x)=\int_{\mathbb{R}^{n}} U(x-\xi) f(\xi) d \xi
$$

whenever $f$ is a continuous function.

REMARK 4.1. If $F \in L^{1}\left(\mathbb{R}^{n}\right) \cap L^{s}\left(\mathbb{R}^{n}\right)$ and $\phi, \psi$ belong to $L^{s}\left(\mathbb{R}^{n}\right), L^{1}\left(\mathbb{R}^{n}\right)$ respectively, then we have that

$$
\phi * F \in L^{s}\left(\mathbb{R}^{n}\right) \text { and } \psi * F \in L^{s}\left(\mathbb{R}^{n}\right) .
$$

Moreover, Young's inequality establishes

$$
\begin{aligned}
& \left\{\int_{\mathbb{R}^{n}}|\phi * F|^{s} d \xi\right\}^{1 / s} \leq\left\{\int_{\mathbb{R}^{n}}|\phi|^{s} d \xi\right\}^{1 / s}\left\{\int_{\mathbb{R}^{n}}|F| d \xi\right\}, \\
& \left\{\int_{\mathbb{R}^{n}}|\psi * F|^{s} d \xi\right\}^{1 / s} \leq\left\{\int_{\mathbb{R}^{n}}|\psi| d \xi\right\}\left\{\int_{\mathbb{R}^{n}}|F|^{s} d \xi\right\}^{1 / s} .
\end{aligned}
$$


In particular, this fact implies

$$
\{\phi+\psi\} * F \in L^{s}\left(\mathbb{R}^{n}\right)
$$

and

$$
\begin{aligned}
& \left\{\int_{\mathbb{R}^{n}}|\{\phi+\psi\} * F|^{s} d \xi\right\}^{1 / s} \\
& \quad \leq\left\{\int_{\mathbf{R}^{n}}|\phi|^{s} d \xi\right\}^{1 / s}\left\{\int_{\mathbb{R}^{n}}|F| d \xi\right\}+\left\{\int_{\mathbf{R}^{n}}|\psi| d \xi\right\}\left\{\int_{\mathbb{R}^{n}}|F|^{s} d \xi\right\}^{1 / s} .
\end{aligned}
$$

LEMMA 4.1. Let us suppose that $v$ is a continuous function satisfying $\partial^{\alpha} v \in L^{p}\left(\mathbb{R}^{n}\right)$ for $|\alpha|=1$; then the following inequality holds:

$$
|v(x)| \leq|v(0)|+8 \frac{\rho}{\rho-2}|x|^{1-2 / \rho}\left(\int_{\mathbf{R}^{n}}|\nabla v|^{p} d \xi\right)^{1 / p} .
$$

Proof. Let us consider the identity

$$
v(\xi)-v(x)=\int_{0}^{1} \nabla v(t \xi+(1-t) x) \cdot[\xi-x] d t .
$$

Integrating it over the ball $B$ of center $x$ and radius $\frac{1}{2}|x-y|$ we get

$$
\begin{aligned}
\left|\int_{B} v(\xi) d \xi-\frac{\pi}{4}\right| x-\left.y\right|^{2} v(x) \mid & \leq \int_{B} \int_{0}^{1}|\nabla v(t \xi+(1-t) x) \cdot[\xi-x]| d t d \xi \\
& \leq|x-y| \int_{B} \int_{0}^{1}|\nabla v(t(\xi-x)+x)| d t d \xi .
\end{aligned}
$$

Introducing the change of variable $\mathbf{y}=t(\xi-x)+x$ we obtain

$$
\left|\int_{B} v(\xi) d \xi-\frac{\pi}{4}\right| x-\left.y\right|^{2} v(x)|\leq| x-y\left|\int_{0}^{1} t^{-2} \int_{t D}\right| \nabla v(\xi) \mid d \xi d t
$$

where $D$ is a disk of center at the origin of coordinates and radius equal to $t|x-y|$. Since $t \leq 1$ and $t D \subseteq D$, it follows that

$$
\int_{t D}|\nabla v(\xi)| d \xi \leq \pi^{1 / p^{\prime}}|x-y|^{2 / p^{\prime}} t^{2 / p^{\prime}}\left(\int_{D}|\nabla v(\xi)|^{p} d \xi\right)^{1 / p},
$$

which together with Eq. (4.2) yields

$$
\left|\int_{B} v(\xi) d \xi-\frac{\pi}{4}\right| x-\left.y\right|^{2} v(x)\left|\leq \pi^{1 / p^{\prime}} \frac{\rho}{\rho-2}\right| x-\left.y\right|^{1+2 / p^{\prime}}\left(\int_{D}|\nabla v(\xi)|^{p} d \xi\right)^{1 / p} .
$$

Interchanging the role of $x$ and $y$ and applying the triangle inequality we obtain

$$
\begin{aligned}
\frac{\pi}{4}|x-y|^{2}|v(x)-v(y)| \leq & \left|\int_{B} v(\xi) d \xi-\frac{\pi}{4}\right| x-\left.y\right|^{2} v(x) \mid \\
& +\left|\int_{B} v(\xi) d \xi-\frac{\pi}{4}\right| x-\left.y\right|^{2} v(y) \mid,
\end{aligned}
$$

from which it follows that

$$
|v(x)-v(y)| \leq 8 \frac{p}{p-2}|x-y|^{1-2 / p}\left(\int_{D}|\nabla u(\xi)|^{p} d \xi\right)^{1 / p} .
$$

Taking $y=0$ and using the triangle inequality the result follows. 
In the following lemma we will establish some regularity properties of the solution of Eq. (4.1).

LEMMA 4.2. Let $f$ be a function satisfying

$$
f \in \mathbf{C}\left(\mathbb{R}^{n}\right) \quad o(f)=o\left(|x|^{-\theta}\right) \quad \text { when }|x| \rightarrow+\infty, \quad \theta>2 \text { for } n=2 ;
$$

then there exists a continuous function $u$ with $\partial^{\alpha} u \in H^{1}\left(\mathbb{R}^{n}\right)$ for $|\alpha|=1$, satisfying Eq. (4.1). Finally if

$$
f \in L^{1}\left(\mathbb{R}^{n}\right) \cap L^{p}\left(\mathbb{R}^{n}\right) \text { for } n \geq 3
$$

where $\rho \in \max \left\{q, q^{\prime}\right\}, q>n /(n-2)$ and $\frac{1}{q}+\frac{1}{q^{\prime}}=1$. There exists a solution $u$ of Eq. (4.1) satisfying $u \in L^{q}\left(\mathbb{R}^{n}\right)$ and $\partial^{\alpha} u \in H^{1}\left(\mathbf{R}^{n}\right),|\alpha|=1$.

Proof. Let us denote by $f_{\nu}$ the following convolution in $\mathbb{R}^{n}, f_{\nu}=\rho_{\nu} * f$, where $\rho_{\nu}$ is the mollifier taken such that

$$
\rho_{\nu}(-x)=\rho_{\nu}(x), \quad \int_{\mathbb{R}^{n}} \rho_{\nu}(\xi) d \xi=1, \quad \text { and } \quad \rho_{\nu}(x)=0 \quad \text { if }|x| \geq \frac{1}{\nu} .
$$

It is well known that $f_{\nu}$ converge to $f$ in $L^{r}$ for any $r \geq 1$ provided $f \in L^{r}$. As we saw above, the sequence $\left(u_{\nu}\right)_{\nu \in \mathbb{N}}$ defined as

$$
u_{\nu}(x)=\int_{\mathbb{R}^{n}} U(x-\xi) f_{\nu}(\xi) d \xi
$$

satisfies the equation

$$
\Delta u_{\nu}=f_{\nu},
$$

from which it easily follows that

$$
\begin{gathered}
\int_{\mathbb{R}^{n}}\left|\nabla u_{\nu}(x)\right|^{2} d x \leq \int_{\mathbb{R}^{n}}\left|u_{\nu}\right|\left|f_{\nu}(x)\right| d x, \\
\int_{\mathbb{R}^{n}}\left|\nabla u_{\nu}(x)-\nabla u_{\mu}(x)\right|^{2} d x \leq \int_{\mathbf{R}^{n}}\left|f_{\nu}(x)-f_{\mu}(x)\right|^{2} d x .
\end{gathered}
$$

From the hypotheses on $f$ we have that if $\left(f_{\nu}\right)_{\nu \in \mathbb{N}}$ is a Cauchy sequence in $L^{2}\left(\mathbb{R}^{n}\right)$ then so is $\left(\Delta u_{\nu}\right)_{\nu \in \mathbb{N}}$. Therefore, in order to prove that $\partial^{\alpha} u \in H^{1}\left(\mathbb{R}^{n}\right)$ for $|\alpha|=1$, we only have to show that $\partial^{\alpha} u \in L^{2}\left(\mathbb{R}^{n}\right)$. To do this it is enough to prove that the right-hand side of Eq. (4.5) is bounded. First we will consider the case $n=2$, for which the derivative of the function $u_{\nu}$ satisfies

$$
\left|\frac{\partial}{\partial x_{i}} u_{\nu}(x)-\frac{\partial}{\partial x_{i}} u_{\mu}(x)\right| \leq \frac{1}{2 \pi} \int_{\mathbf{R}^{n}}|x-\xi|^{-1}\left|f_{\nu}(\xi)-f_{\mu}(\xi)\right| d \xi .
$$

The hypotheses on $f$ imply that $f \in L^{r}\left(\mathbb{R}^{n}\right) \quad \forall r \geq 1$. Since $\theta>2$, there exists $\rho>2$ such that $\theta-(1-2 / p)>2$. Applying Remark 4.1 for $\psi=\chi|\xi|^{-1}, \phi=\chi_{\mathrm{c}}|\xi|^{-1}$, $F=f_{\nu}-f_{\mu}$, and $s=\rho$, where $\chi$ and $\chi_{c}$ stands for the characteristic function on the open ball $B(0,1)$ and its complementary set, respectively, we get

$$
x \mapsto \int_{\mathbb{R}^{n}}|x-\xi|^{-1}\left|f_{\nu}(\xi)-f_{\mu}(\xi)\right| d \xi \in L^{p}\left(\mathbb{R}^{n}\right) ;
$$


therefore,

$$
\frac{\partial}{\partial x_{i}} u_{\nu}(x) \in L^{p}\left(\mathbb{R}^{n}\right) \quad \forall \nu \in \mathbb{N}
$$

and

$$
\begin{aligned}
\left\{\int_{\mathbf{R}^{n}}\left|\frac{\partial}{\partial x_{i}} u_{\nu}(x)-\frac{\partial}{\partial x_{i}} u_{\mu}(x)\right|^{p} d x\right\}^{1 / p} \leq & \frac{1}{2 \pi} \int_{\mathbb{R}^{n}} \chi|\xi|^{-1} d x\left\{\int_{\mathbb{R}^{n}}\left|f_{\nu}-f_{\mu}\right|^{p} d x\right\}^{1 / p} \\
& +\left\{\frac{1}{2 \pi} \int_{\mathbf{R}^{n}} \chi_{\mathbf{c}}|\xi|^{-p} d x\right\}^{1 / p} \int_{\mathbb{R}^{n}}\left|f_{\nu}-f_{\mu}\right| d x
\end{aligned}
$$

hence, $\left(\partial u_{\nu} / \partial x_{i}\right)_{\nu \in \mathbb{N}}$ is a Cauchy sequence in $L^{p}\left(\mathbb{R}^{n}\right)$ and so is bounded. Applying Lemma 4.1 to the Cauchy difference $u_{\nu}(x)-u_{\mu}(x)$ we obtain

$$
\left|u_{\nu}(x)-u_{\mu}(x)\right| \leq\left|u_{\nu}(\mathbf{0})-u_{\mu}(\mathbf{0})\right|+C|x|^{\alpha}\left\{\int_{\mathbb{R}^{2}}\left|\nabla u_{\nu}(\xi)-\nabla u_{\mu}(\xi)\right|^{p} d \xi\right\}^{1 / p}
$$

where $a=1-2 / \rho$. In order to prove that $\left(u_{\nu}\right)_{\nu \in \mathbb{N}}$ is a Cauchy sequence in $\mathbf{C}$ we only need to show that the numerical sequence $\left(u_{\nu}(\mathbf{0})\right)_{\nu \in \mathbb{N}}$ is convergent. But this follows immediately from the definition of $u_{\nu}$ and the hypotheses on $f$; so there exists a continuous function $u$ such that

$$
\begin{aligned}
u_{\nu} & \rightarrow u \text { uniformly on bounded sets of } \mathbb{R}^{2}, \\
\partial^{\alpha} u_{\nu} & \rightarrow \partial^{\alpha} u \text { strongly in } L^{p}\left(\mathbb{R}^{2}\right), \rho>2,|\alpha|=1 .
\end{aligned}
$$

Lemma 4.1 implies that

$$
\left|u_{\nu}(x)\right| \leq\left|u_{\nu}(\mathbf{0})\right|+\frac{8 \rho|x|^{1-2 / p}}{\rho-2}\left(\int_{\mathbb{R}^{n}}\left|\nabla u_{\nu}(\xi)\right|^{p} d \xi\right)^{1 / p} .
$$

Since $o(f)=o\left(|x|^{-\theta}\right)$, it follows that $o\left(f_{\nu}\right)=o\left(|x|^{-\theta}\right)$ (consider the identity

$$
f_{\nu}(x)=\int_{|\xi| \leq \frac{1}{\nu}} \rho_{\nu}(\xi) f(x-\xi) d \xi
$$

and apply the hypotheses on $f$ ). Therefore, we have

$$
o\left(f_{\nu} u_{\nu}\right)=o\left(|x|^{-\theta+\alpha}\right) \text { when } x \rightarrow \infty,
$$

which implies that $f_{\nu} u_{\nu}$ is bounded in $L^{1}\left(\mathbb{R}^{n}\right)$ for any $\nu \in \mathbb{N}$. Hence, the righthand side of Eq. (4.5) is bounded. Then our result follows. Let us consider the case $n>2$. We will prove that $\left(\partial^{\alpha} u_{\nu}\right)_{\nu \in \mathbb{N}}$ is bounded in $H^{1}\left(\mathbb{R}^{n}\right)$ for $|\alpha|=1$. In fact, since $\chi U$ and $\chi_{\mathrm{c}} U$ belong to $L^{1}\left(\mathbb{R}^{n}\right)$ and $L^{q}\left(\mathbb{R}^{n}\right)$ where $q>n /(n-2)$, applying Remark 4.1 for $\psi=\chi U, \phi=\chi_{\mathrm{c}} U, s=q$, and $F=f_{\nu}$ yields

$$
\begin{aligned}
{\left[\int_{\mathbb{R}^{n}}\left|u_{\nu}(x)-u_{\mu}(x)\right|^{q} d x\right]^{1 / q} \leq } & C \int_{\mathbb{R}^{n}}\left|\chi_{\mathrm{c}} U\right| d x\left[\int_{\mathbf{R}^{n}}\left|f_{\nu}-f_{\mu}\right|^{q} d x\right]^{1 / q} \\
& +C\left[\int_{\mathbb{R}^{n}}\left|\chi_{\mathrm{c}} U\right|^{q} d x\right]^{1 / q} \int_{\mathbb{R}^{n}}\left|f_{\nu}-f_{\mu}\right| d x .
\end{aligned}
$$


Hence, $\left(u_{\nu}\right)_{\nu \in \mathbb{N}}$ is a Cauchy sequence in $L^{q}\left(\mathbb{R}^{n}\right)$; so we have that there exists $u$ in $L^{q}$ such that

$$
u_{\nu} \rightarrow u \text { strongly in } L^{q}\left(\mathbb{R}^{n}\right) .
$$

Since $f_{\nu}$ converges to $f$ in $L^{q^{\prime}}\left(\mathbb{R}^{n}\right)$, we conclude that the product $f_{\nu} u_{\nu}$ converges to $f u$ in $L^{1}\left(\mathbb{R}^{n}\right)$; then Eq. (4.5) implies

$$
\partial^{\alpha} u_{\nu} \rightarrow \partial^{\alpha} u \text { strongly in } L^{2}\left(\mathbb{R}^{n}\right),|\alpha|=1 .
$$

The proof is now complete.

Lemma 4.3. Let $\mathbf{F}$ be a vector field in $\left[H^{k}\left(\mathbb{R}^{n}\right)\right]^{n}$ such that the divergence of $\mathbf{F}$ $(\operatorname{div} \mathbf{F}=f)$ satisfies the conditions of Lemma 4.1 . Then we can decompose $\mathbf{F}$ into two parts, both in $\left[H^{k}\left(\mathbb{R}^{n}\right) j^{n}\right.$, one of them a gradient and the other a solenoidal function (that is, with null divergence).

Proof. From Lemma 4.2 there exists a function $\rho$ such that $\partial^{\alpha} \rho \in H^{1}\left(\mathbb{R}^{n}\right)$ for $|\alpha|=1$, satisfying

$$
\Delta \rho=\operatorname{div} \mathbf{F} \text { in } \mathbb{R}^{n} .
$$

Since $\Delta \rho=\operatorname{div} \mathbf{F} \in H^{k-1}\left(\mathbb{R}^{n}\right)$, we have that $\partial^{\alpha} \rho \in H^{k}\left(\mathbb{R}^{n}\right)$ for any $|\alpha|=1$, and from the identity $\mathbf{F}=\nabla \rho+(\mathbf{F}-\nabla \rho)$ we obtain the desired decompositions.

LEMMA 4.4. Let us denote

$$
\mathbf{u}_{0}=\left(u_{0}^{1}, \ldots, u_{0}^{n}\right), \quad \mathbf{u}_{1}=\left(u_{1}^{1}, \ldots, u_{1}^{n}\right) ;
$$

then if the initial data $u_{0}$ and $u_{1}$ are such that

$$
D_{i} u_{0}^{j}(x)=D_{j} u_{0}^{i}(x), \quad D_{i} u_{1}^{j}(x)=D_{j} u_{1}^{i}(x) \quad \text { for } i \neq j,
$$

the solution $\mathbf{u}$ of system (1.1) also satisfies

$$
D_{i} u^{j}(\mathbf{x}, t)=D_{j} u^{i}(\mathbf{x}, t), \quad D_{i} u_{t}^{j}(\mathbf{x}, t)=D_{j} u_{t}^{i}(\mathbf{x}, t) \quad \text { in } \mathbb{R}_{x}^{n} \times \mathbb{R}_{t} .
$$

Moreover, if $\operatorname{div} \mathbf{u}_{0}=\operatorname{div} \mathbf{u}_{1}=\mathbf{0}$, then $\mathbf{u}$ is a solenoidal function.

Proof. Denoting $\partial_{i}=\partial^{\alpha}$ for $\alpha=(0, \ldots, 1, \ldots, 0)$, where the 1 is in the $i$ th position, from system (1.1) it is easy to see that

$$
C_{i j} \mathbf{u} \stackrel{\text { def }}{=} \partial_{i} u^{j}-\partial_{j} u^{i}
$$

satisfies

$$
\begin{gathered}
\left\{C_{i j} \mathbf{u}\right\}_{t t}-\Delta\left\{C_{i j} \mathbf{u}\right\}+g * \Delta\left\{C_{i j} \mathbf{u}\right\}=0, \\
C_{i j} \mathbf{u}(\mathbf{x}, 0)=0, \quad C_{i j} \mathbf{u} t(\mathbf{x}, 0)=0 ;
\end{gathered}
$$

on the other hand, the divergence operator solves

$$
\begin{gathered}
\{\operatorname{div} \mathbf{u}\}_{t t}-\Delta\{\operatorname{div} \mathbf{u}\}+g * \Delta\{\operatorname{div} \mathbf{u}\}=0, \\
\operatorname{div} \mathbf{u}(\mathbf{x}, 0)=0, \quad \operatorname{div} \mathbf{u}_{t}(\mathbf{x}, 0)=0 .
\end{gathered}
$$

By the uniqueness of the solution our result follows.

From Lemma 4.3 we can decompose the initial data into two parts, say

$$
\mathbf{u}_{0} \equiv \mathbf{u}_{0}^{s}+\mathbf{u}_{\mathbf{c}}^{i}, \quad \mathbf{u}_{1} \equiv \mathbf{u}_{1}^{s}+\mathbf{u}_{1}^{i}
$$


where $\operatorname{div}\left(\mathbf{u}_{0}^{s}\right)=0, \operatorname{div}\left(\mathbf{u}_{1}^{s}\right)=0$, and $\mathbf{u}_{\mathbf{c}}^{i}=\nabla \rho_{0}, \mathbf{u}_{1}^{i}=\nabla \rho_{1}$ for some functions $\rho_{0}, \rho_{1}$. Let us denote by $\mathbf{u}^{s}$ the solution of the system

$$
\begin{gathered}
\mathbf{u}_{t t}^{s}-\mu \Delta \mathbf{u}^{s}+g * \Delta \mathbf{u}^{s}=0 \quad \text { in } \mathbb{R}_{x}^{n} \times \mathbb{R}_{t}, \\
\mathbf{u}^{s}(0)=\mathbf{u}_{0}^{s} \text { and } \mathbf{u}_{t}^{s}(0)=\mathbf{u}_{1}^{s} \quad \text { in } \mathbb{R}_{x}^{n} .
\end{gathered}
$$

From Lemma 4.4 we conclude that

$$
\operatorname{div} \mathbf{u}^{s}=\operatorname{div} \mathbf{u}_{t}^{s}=0 \text { in } \mathbb{R}_{x}^{n} .
$$

On the other hand, if $\mathbf{u}^{i}$ is the solution of

$$
\begin{gathered}
\mathbf{u}_{t t}^{i}-(\lambda+\mu) \mathbf{u}^{i}+h * \Delta \mathbf{u}^{i}=0 \quad \text { in } \mathbb{R}_{x}^{n} \times \mathbb{R}_{t}, \\
\mathbf{u}^{i}(0)=\mathbf{u}_{\mathbf{c}}^{i} \text { and } \mathbf{u}_{t}^{i}(0)=\mathbf{u}_{1}^{i} \text { in } \mathbb{R}_{x}^{n},
\end{gathered}
$$

Lemma 4.4 implies that $C_{i j} \mathbf{u}^{s}=0$; therefore, we can express $\mathbf{u}^{i}=\nabla \rho$ for some scalar function $\rho$. Note that in this case we have

$$
\nabla \operatorname{div} \mathbf{u}^{i}=\nabla\{\Delta \rho\}=\Delta\{\nabla \rho\}=\Delta \mathbf{u}^{i} ;
$$

therefore, the function $\mathbf{u}=\mathbf{u}^{s}+\mathbf{u}^{i}$ satisfies Eq. (1.1). By the symmetry of the system (4.7) and (4.8) it is sufficient to study one of them; therefore, denoting $\mathbf{u}=\mathbf{u}^{s}$, $\mathbf{u}_{0}=\mathbf{u}_{0}^{s}$, and $\mathbf{u}_{1}=\mathbf{u}_{1}^{s}$, we will consider

$$
\begin{gathered}
\mathbf{u}_{t t}-\mu \Delta \mathbf{u}+g * \Delta \mathbf{u}=0 \quad \text { in } \mathbb{R}_{x}^{n} \times \mathbb{R}_{t}, \\
\mathbf{u}(0)=\mathbf{u}_{0} \text { and } \mathbf{u}_{t}(0)=\mathbf{u}_{1} \quad \text { in } \mathbb{R}_{x}^{n} .
\end{gathered}
$$

In this case we will define the energy functions as

$$
\begin{aligned}
& E_{1}(t)=\frac{1}{2} \int_{\Omega}\left\{\left|\mathbf{u}_{t}\right|^{2}+\mu \sum_{i=1}^{n}\left(1-\int_{0}^{t} g d \tau\right)\left|\nabla u_{i}\right|^{2}\right\} d x+\frac{\mu}{2} \sum_{i=1}^{n} \int_{\Omega} g \square \nabla u_{i} d x, \\
& E_{2}(t)=\frac{1}{2} \int_{\Omega}\left\{\sum_{i=1}^{n}\left|\nabla u_{i, t}\right|^{2}+\mu\left(1-\int_{0}^{t} g d \tau\right)|\Delta \mathbf{u}|^{2}\right\} d x+\frac{\mu}{2} \sum_{i=1}^{n} \int_{\Omega} g \square \Delta \mathbf{u} d x,
\end{aligned}
$$

and

$$
E_{3}(t)=\frac{1}{2} \int_{\Omega}\left\{\left|\mathbf{u}_{t t}\right|^{2}+\mu \sum_{i=1}^{n}\left|\nabla u_{i, t}\right|^{2}\right\} d x .
$$

We will conclude the asymptotic behaviour of the energy by studying the asymptotic properties of the energy density function given by

$$
\begin{aligned}
\mathscr{E}_{1}(\xi, t) & \stackrel{\text { def }}{=} \frac{1}{2}\left|\hat{\mathbf{u}}_{t}\right|^{2}+\mu|\xi|^{2}\left(1-\int_{0}^{t} g d \tau\right) \sum_{i=1}^{n}\left|\hat{u}_{i}\right|^{2}+\frac{\mu}{2}|\xi|^{2} \sum_{i=1}^{n} g \square \hat{u}_{i}, \\
\mathscr{E}_{2}(\xi, t) & \stackrel{\text { def }}{=}|\xi|^{2} \mathscr{E}_{1}(\xi, t), \\
\mathscr{E}_{3}(\xi, t) & \stackrel{\text { def }}{=}\left|\hat{\mathbf{u}}_{t t}\right|^{2}+\mu \sum_{i=1}^{n}|\xi|^{2}\left|\hat{u}_{i, t}\right|^{2} \\
i(t) & \stackrel{\text { def }}{=} \operatorname{Re}\left\{\hat{\mathbf{u}}_{t t} \cdot \overline{\hat{\mathbf{u}}}_{t}\right\}+\frac{1}{2} g(0)\left|\hat{\mathbf{u}}_{t}\right|^{2}+\frac{\mu}{2}|\xi|^{2} \sum_{i=1}^{n} f \square \hat{u}_{i}-\frac{\mu}{2}\left(\int_{0}^{t} f d \tau\right) \sum_{i=1}^{n}\left|\hat{u}_{i}\right|^{2}
\end{aligned}
$$


and

$$
\mathscr{F}(t) \stackrel{\text { def }}{=} \mu|\xi|^{2} \operatorname{Re}\left\{\hat{\mathbf{u}}_{t} \cdot \overline{\hat{\mathbf{u}}}\right\}
$$

where $\hat{v}$ denotes the usual Fourier transform of $v$.

To conclude that the rate of decay depends on the regularity of the initial data, we will use the following lemmas.

LeMma 4.5. Let $v$ be a function in $L^{2}\left(\mathbb{R}^{n}\right) \cap L^{1}\left(\mathbb{R}^{n}\right)$ for which there exist $f \in$ $L^{2}\left(\mathbb{R}^{n}\right) \cap L^{1}\left(\mathbb{R}^{n}\right)$ satisfying

$$
v=\partial^{\alpha} f \quad \text { where }|\alpha|=m .
$$

Then we have

$$
|\hat{v}(\xi)| \leq[2 \pi]^{-n / 2}\left|\xi^{\alpha}\right| \int_{\mathbb{R}^{n}}|f(x)| d x \quad \forall \xi \in \mathbb{R}^{n} .
$$

Proof. Integration by parts of $\hat{v}$ implies that

$$
\hat{v}(\xi)=[2 \pi]^{-n / 2}(-i)^{|\alpha|}\left|\xi^{\alpha}\right| \int_{\mathbb{R}^{n}} f(x) e^{i x \xi} d x
$$

where $i=\sqrt{-1}$. Since $f \in L^{2}\left(\mathbb{R}^{n}\right) \cap L^{1}\left(\mathbb{R}^{n}\right)$ our result follows.

LEMMA 4.6. If $r$ is a positive real number, then we have

$$
\int_{0}^{1} \eta^{2 m} e^{-r \eta^{2}} d \eta \leq(2 m) ! \sqrt{\pi / 2} r^{-m-1 / 2}, \quad m=0,1, \ldots
$$

Proof. Let us denote by $I_{m}$ the integral in the left-hand side of the above inequality. Straightforward calculations give the inequality

$$
I_{0}=\int_{0}^{1} e^{-r \eta^{2}} d \eta \leq \sqrt{\pi / 2} r^{-1 / 2} .
$$

Reiterated use of integration by parts on $I_{m}$ with $m>0$ implies

$$
I_{m} \leq(2 r)^{-1}(2 m-1) \int_{0}^{1} \eta^{2 m-2} e^{-r \eta^{2}} d \eta \leq(2 r)^{-1}(2 m-1) I_{m-1} \leq r^{-m}(2 m) ! I_{0}
$$

which together with Eq. (4.9) yields the required inequality.

Let us denote by $E_{k}^{i}$ and $E_{k}^{s}$ the energies stored in the irrotational and the solenoidal parts of the displacement vector field, respectively, for $k=1,2,3$. We will express our final result in the following theorem.

THEOREM 4.1. Let us suppose $f, g$ are $C^{2}$-functions satisfying Eqs. (1.4), (1.5), and (2.3). Let $\mathbf{u}_{0}$ and $\mathbf{u}_{1}$ be vector fields in $\left[H^{2}\left(\mathbb{R}^{n}\right)\right]^{n}$ and $\left[H^{1}\left(\mathbb{R}^{n}\right)\right]^{n}$ respectively, such that $\operatorname{div} \mathbf{u}_{0}$ and div $\mathbf{u}_{1}$ satisfy the hypotheses of Lemma 4.3. Then the displacement vector field can be decomposed into two parts, one of them solenoidal and the other a gradient whose associated energies decay as

$$
\begin{aligned}
& E_{1}^{i}(t)+E_{2}^{i}(t)+E_{3}^{i}(t) \leq c_{1} t^{-n / 2}, \\
& E_{1}^{s}(t)+E_{2}^{s}(t)+E_{3}^{s}(t) \leq c_{1} t^{-n / 2} .
\end{aligned}
$$


Moreover, if there exist functions $f_{0}^{k}$ and $f_{1}^{k} \in L^{2}\left(\mathbb{R}^{n}\right) \cap L^{1}\left(\mathbb{R}^{n}\right)$ such that

$$
\partial^{\alpha_{k}} f_{\alpha}^{k}=u_{0}^{k} \quad \text { and } \quad \partial^{\beta_{k}} f_{1}^{k}=u_{1}^{k}, \quad k=1, \ldots, n,
$$

then

$$
\begin{aligned}
& E_{1}^{i}(t)+E_{2}^{i}(t)+E_{3}^{i}(t) \leq c_{1} t^{-m-n / 2}, \\
& E_{1}^{s}(t)+E_{2}^{s}(t)+E_{3}^{s}(t) \leq c_{1} t^{-m-n / 2}
\end{aligned}
$$

where $m=\min \left\{\left|\alpha_{k}\right|+1,\left|\beta_{k}\right| ; k=1, \ldots, n\right\}$.

Proof. We will suppose that $\left(\mathbf{u}_{0}, \mathbf{u}_{1}\right) \in D\left(A^{2}\right) \times D(A)$. Our conclusion will follow from the fact that $D\left(A^{2}\right) \times D(A)$ is dense in $\left[H^{2}\left(\mathbb{R}^{n}\right)\right]^{n} \times\left[H^{1}\left(\mathbb{R}^{n}\right)\right]^{n}$. In view of the above discussion it is sufficient to prove the result for $\mathbf{u}=\mathbf{u}^{i}$. Let us introduce the following function:

$$
\begin{aligned}
\mathscr{K}(t) & =\mathscr{E}_{3}(t)+g(0)\left\{1-\frac{g(0)}{4 \kappa}\right\} i(t)+g(0)\left\{1-\frac{g(0)}{2 \kappa}\right\} \mathscr{K}(t)+\mathscr{N}_{1} \mathscr{E}_{1}(t)+\mathscr{N}_{2} \mathscr{E}_{2}(t), \\
\mathscr{R} & =\left|\hat{\mathbf{u}}_{t}\right|^{2}+\mu|\xi|^{2} \sum_{i=1}^{n}\left|\hat{u}_{i}\right|^{2},
\end{aligned}
$$

and

$$
\mathscr{M}=\left|\hat{\mathbf{u}}_{t t}\right|^{2}+\mu \sum_{i=1}^{n}|\xi|^{2}\left|\hat{u}_{i, t}\right|^{2}+\mu|\xi|^{4}|\mathbf{u}|^{2}+\mu\left\{|\xi|^{4}+|\xi|^{2}\right\} \sum_{i=1}^{n} g \square \hat{u}_{i} .
$$

As in the proof of Theorem 3.1 we conclude that

$$
\frac{d}{d t} \mathscr{K}(\xi, t) \leq-\alpha \frac{g(0)^{2}}{4 \kappa} \mathscr{M}(\xi, t)
$$

and

$$
\mathscr{K}(\xi, t) \leq c_{1} \mathscr{M}+(2+g(0)+\alpha) \mathscr{R}(\xi, t) .
$$

In order to prove the uniform decay of $\mathbf{u}$, we will consider two cases. First we will prove that the energy density decays exponentially for $|\xi| \geq 1$ and then that the uniform decay holds for $|\xi| \leq 1$. In fact, for $|\xi| \geq 1$ we have

$$
\mathscr{K}(\xi, t) \leq\left\{c_{1}+2+g(0)+\alpha\right\} \mathscr{M} \text { for }|\xi| \geq 1
$$

which together with Eqs. (4.10) and (4.11) yields the inequality

$$
\frac{d}{d t} \mathscr{K}(\xi, t)+\gamma \mathscr{K}(\xi, t) \leq 0
$$

for $\gamma=\alpha g(0)^{2} /\left(4 \kappa c_{2}\right)$, where $c_{2}=\left\{c_{1}+2+g(0)+\alpha\right\}$, from which we get

$$
\mathscr{K}(\xi, t) \leq \mathscr{K}(\xi, 0) e^{-\gamma t} .
$$

Integrating over $|\xi| \geq 1$, we obtain

$$
\int_{|\xi| \geq 1} \mathscr{K}(\xi, t) d \xi \leq\left\{\int_{|\xi| \geq 1} \mathscr{K}(\xi, 0) d \xi\right\} e^{-\gamma t} .
$$

On the other hand, since

$$
|\xi|^{2} \mathscr{K}(\xi, t) \leq\left(c_{1}+2+g(0)+\alpha\right) \mathscr{M} \quad \forall|\xi| \leq 1,
$$


inequalities (4.10) and (4.13) imply

$$
\frac{d}{d t} \mathscr{K}(\xi, t)+\gamma|\xi|^{2} \mathscr{K}(\xi, t) \leq 0 ;
$$

so we have

$$
\mathscr{K}(\xi, t) \leq \mathscr{K}(\xi, 0) e^{-\gamma|\xi|^{2} t} .
$$

Integrating the above inequality over $|\xi| \leq 1$ and using Lemma 4.6 we conclude that there exists a positive constant $C_{1}$ such that

$$
\int_{|\xi| \leq 1} \mathscr{K}(\xi, t) d \xi \leq C_{1}\left\{\int_{|\xi| \leq 1} \mathscr{K}(\xi, 0) d \xi\right\} t^{-n / 2} .
$$

From Eq. (4.12) and Plancherel's identity, part (i) follows. Finally to prove part (ii), let us suppose that there exist functions $f_{0}^{k}, f_{1}^{k} \in L^{2}\left(\mathbb{R}^{n}\right) \cap L^{1}\left(\mathbb{R}^{n}\right)$ satisfying the hypotheses of this theorem; then there exists $C_{2}>0$ satisfying

$$
\mathscr{K}(\xi, 0) \leq C_{2}\left\{|\xi|^{2}\left|\hat{\mathbf{u}}_{1}(\xi)\right|^{2}+|\xi|^{4}\left|\hat{\mathbf{u}}_{0}(\xi)\right|^{2}\right\} .
$$

From Lemma 4.5 we have that

$$
\mathscr{K}(\xi, 0) \leq C_{3}\left\{\sum_{k=1}^{n}\left|\xi^{\alpha_{k}}\right|+|\xi|^{2} \sum_{k=1}^{n}\left|\xi^{\beta_{k}}\right|\right\}
$$

where

$$
C_{3}=\max \left\{\int_{\mathbb{R}^{n}}\left|f_{0}^{k}\right| d \xi, \int_{\mathbb{R}^{n}}\left|f_{1}^{k}\right| d \xi ; k=1, \ldots, n\right\} .
$$

Since

$$
\begin{array}{rl}
\int_{|\xi| \leq 1} & \mathscr{K}(\xi, 0) e^{-\gamma|\xi|^{2} t} \\
\quad \leq C_{4} \int_{|\xi| \leq 1}\left\{\sum_{k=1}^{n}\left|\xi^{\alpha_{k}}\right|+|\xi|^{2} \sum_{k=1}^{n}\left|\xi^{\beta_{k}}\right|+\sum_{k=1}^{n}\left|\xi^{\alpha}\right|\right\} e^{-\gamma|\xi|^{2} t} d \xi,
\end{array}
$$

by iterated integration and applying Lemma 4.6 we conclude that

$$
\int_{|\xi| \leq 1} \mathscr{K}(\xi, 0) e^{-\gamma|\xi|^{2} t} \leq C_{5} t^{-m-n / 2}
$$

for $C_{5}>0$. As in the proof of Theorem 3.1 we have

$$
\int_{\mathbb{R}^{n}} \mathscr{K}(\xi, t) d \xi \geq c_{0} \int_{\mathbb{R}^{n}} \mathscr{M}(\xi, t)+\mathscr{R}(\xi, t) d \xi .
$$

From Eqs. (4.12) and (4.14) and the Plancherel identity our result follows.

\section{REFERENCES}

[1] G. Creus, Viscoelasticity basic theory and applications to concrete structures, (C. A. Brebbia and S. A. Orszag, eds.), Lecture Notes in Engineering, vol. 16, Springer-Verlag, New York, 1986

[2] C. M. Dafermos, An abstract Volterra equation with applications to linear viscoelasticity, J. Differential Equations 7, 554-569 (1970)

[3] C. M. Dafermos, Asymptotic stability in viscoelasticity, Arch. Rational Mech. Anal. 37, 297-308 (1970) 
[4] C. M. Dafermos, Energy methods for nonlinear hyperbolic Volterra integrodifferential equations, Comm. Partial Differential Equations (3), 178-219 (1979)

[5] G. Dassios and F. Zafiropoulos, Equipartition of energy in linearized 3-D viscoelasticity, Quart. Appl. Math. 48, 715-730 (1990)

[6] J. L. Lions, Quelques méthodes de résolution de problèmes aux limites non lineares, Dunod Gauthier Villars, Paris, 1969 